\title{
Spinal Epidural Capillary Hemangioma: a Review
}

\author{
Frederico de Lima Gibbon ${ }^{1 *}$, Guilherme Gago da Silva ${ }^{2}$ \\ ${ }^{1}$ Hospital, Complexo Hospitalar Santa Casa de Misericórdia de Porto Alegre, Porto Alegre - Brazil. \\ ${ }^{2}$ School of Medicine, Universidade Católica de Pelotas, and Pelotas - Brazil. \\ * Corresponding Author: Frederico de Lima Gibbon. School of Medicine, Universidade Católica de Pelotas, Pelotas Brazil. \\ Received date: April 08, 2019; Accepted date: May 07, 2019; Published date: May 14, 2019. \\ Citation: Frederico de Lima Gibbon, and Guilherme Gago da Silva. (2019) Spinal Epidural Capillary Hemangioma: a Review, J. Neuroscience and \\ Neurological Surgery, 4(2); Doi:10.31579/2578-8868/155
}

Copyright: (c) 2019 Frederico de Lima Gibbon. This is an open-access article distributed under the terms of The Creative Commons Attribution License, which permits unrestricted use, distribution, and reproduction in any medium, provided the original author and source are credited.

\section{Abstract}

The hemangiomas are benign vascular malformations. There are two types of hemangiomas that may affect the central nervous system, the cavernous type and the capillary type. The last is most uncommon and its presentation at spinal epidural space is extremely rare. These lesions usually present with signs and symptoms of myelopathy, radiculopathy or cauda equine syndrome. Magnetic resonance imaging (MRI) is the examination of choice and the treatment must be surgical regardless of the presentation of the patient due to the risk of spinal cord compression.

Keywords: capillary hemangioma, epidural vascular malformation, spinal capillary, spinal hemangioma, epidural hemangioma.

\section{Introduction:}

Hemangiomas are benign vascular malformations, which pathologists usually define as hamartomas. There are two large groups according to histology, being capillary and cavernous $[1,2]$. In the central nervous system (CNS), the most common type is the cavernous [3,4]. The capillary type typically presents in places like skin, bones and subcutaneous, being very rare its presentation in the CNS, especially in the vertebral column [2,5]. Hemangiomas affecting the spine are commonly seen in the vertebral bodies. Intradural lesions, whether or not affecting the spinal cord, are uncommon, and purely epidurallesions are very rare. The histology, clinical presentation, evaluation and management for spinal epidural capillary hemangioma are presented here.

\section{Histology and clinical presentation:}

CNS capillary hemangiomas are histologically identical to capillary hemangiomas from other sites of the body. The capillary hemangioma consist of numerous thin irregular capillary-sized vessels that are lined by either plump or flattened endothelium and caught in lobular architecture and the presence of a lining of a continuous basal lamina. Whilst cavernous hemangiomas consist of dilated hyaline vessels and often show thrombosis, perivascular hemosiderin deposition, and calcification $[2,5,6]$.

The histological aspects are important to understand the presentantion of both capillary and cavernous. The typical presentation of capillary hemangiomas is as a mass effect since they have a well-defined locular architecture. In contrast to cavernous hemangiomas which presents classicaly an apopletic event due to its capillaries highlypermeable and susceptible to thrombosis $[2,4]$.

Spinal epidural capillary hemangioma normally arises as an extension of a vertebral hemangioma which grows through spinal canal, being extremely rare a purely epidural capillary hemangioma. Spinal epidural capillary hemangioma may arise anywhere along the spinal canal, including cervical, thoracic and cauda equine. Therefore they occur more commonly in the thoracic spine [7], it seems occur just in consequence of thoracic segment be the most longer.

As the capillary hemangioma usually appears at the thoracic segment and presents as a mass effect, the most common presentation is chronic myelopathy, and back pain $[3,7,10]$. When the lesion occurs atcauda equine or extends through the foramen, it may present as cauda equine syndrome or radiculopathy $[5,7,11]$.
In summary, the capillary hemangioma may present as any other epidural tumor and its signs and symptoms are due its location.

\section{Evaluation and management:}

The main exam to investigate the suspected capillary hemangioma is MRI, which is useful for stating the diagnosis, ruling out other hypotheses, and planning treatment [12].

The characteristic findings of capillary hemangiomas in MRI do not differ from capillary hemangiomas from other locations [13,14]. At thepathologic examination, the lesion shows capillary vessels caught in low- attenuating fibrosis without fat or hemosiderin componentes which is reflected in the MRI as homogeneous aspect and the isointense signal relative to the spinal cord on T1-weighted images, hyperintense on T2- weighted images, and as homogeneous enhancement on contrast-enhancedT1-weighted images [13]. Also the rim of low signal intensity seen on T1- and T2-weighted MRI images may correspond with the encapsulated aspecto of the lesion [3].

Nevertheless, the radiological appearance is non-specific, making the differential diagnosis vast. In this case tumors such as nerve sheath tumors, meningiomas, hemangioblastomas, hemangiopericytomas and lymphomas must always be remembered [9]. Capillary hemangiomas tend to have a foraminal extension leading to the dumbbell shape on MRI, this feature differentiates them from cavernomas, although it is classicallyattributed to schwannomas and meningiomas. As a result they shouldalways be included in the differential diagnosis $[3,14,45]$.

Surgery is the treatment of choice. Gross total resection should be the goal as the lesions is benign and completely removal may cure $[2,8,12]$. Although the literature lack of evidence, it seems to be a consensus that the surgical resection of the lesion should be made independent of the clinical presentation at least, considering the risk of spinal cord compression[1,2,10,15]

The most common approach is through a laminectomy/laminotomy aiming en bloc removal of the lesion. In view of the capillary hemangioma be a very vascularized lesion, the piece-to-piece removal may promote considerable bleeding at the site which may became difficult to remove the lesion completely [11]. In addition, another factor that may hinder the total removal of the lesion is the foraminal extension [3]. During the procedure, the lesion may be seen as reddish epidural mass with arterial feeders surrounding it [1].In some cases angiography and preoperative embolization may be useful[1,11]. 
Xu W. Chuanping Gao,Ji Qing Lian Duan Feng,and Jiu Fa Cui, et al. (2017). Spinal Epidural Cavernous Hemangioma: A Clinical Series of 7 Patients. World Neurosurg. (111): 183-191.

7. Brasil A B, Rohrmoser R, Gago G. and Cambruzzi E. (2018). Atypical spinal epidural capillary hemangioma: Case report. Surg. Neurol. Int. (9): 198.

8. Alakandy L. M. (2006). Thoracic intradural extramedullarycapillary haemangioma. Br. J. Neurosurg. (20): 235-238.

9. Rajeev M, Waykule P, Pavitharan V. and Nandeesh B. (2017). Spinal epidural capillary hemangioma: A rare case report with a review of literature. Surg. Neurol. Int. (8): 123.

10. Tekin T. Bayrakli Fatih, Simsek Hakan, Colak Ahmet, and Kutlay Murat,et al. (2008). Lumbar epidural capillary hemangioma presenting as lumbar disc herniation disease: Case report. Spine (Phila. Pa. 1976). (33): 795-797.

11. Cofano, F. Nicola Marengo, Federico Pecoraro, Federica Penner, and Luca Bertero ORCID Icon. et al. (2019). Spinal epidural capillary hemangioma: case report and review of the literature. $\mathrm{Br}$. J. Neurosurg. (0): 1-4.

12. Ji Hoon Shin, Ho Kyu Lee, Sang Ryong Jeon, S. H. P. (2000). Spinal Intradural Capillary Hemangioma: MR Findings. AJNR Am J Neuroradiol 21, 954-956.

13. Gupta S, Kumar S, Banerji D, Pandey R. and Gujral R. et al. epidural spinal haemangiorna. (2):342-344.

14. Hasan A, Guiot, M C, Torres C. and Marcoux J. (2011). A case of a spinal epidural capillary hemangioma: Case report. Neurosurgery (68):850-853. 